\title{
Digital signals processing using non-linear orthogonal transformation in frequency domain
}

\begin{abstract}
The rapid progress of computer technology in recent decades led to a wide introduction of methods of digital information processing practically in all fields of scientific research. In this case, among various applications of computing one of the most important places is occupied by digital processing systems signals (DSP) that are used in data processing remote solution tasks of navigation of aerospace and marine objects, communications, radiophysics, digital optics and in a number of other applications.

Digital Signal Processing (DSP) is a dynamically developing an area that covers both technical and software tools. Related areas for digital signal processing are theory information, in particular, the theory of optimal signal reception and theory pattern recognition. In the first case, the main problem is signal extraction against a background of noise and interference of a different physical nature, and in the second - automatic recognition, i.e. classification and signal identification. In the digital processing of signals under a signal, we mean its mathematical description, i.e. a certain real function, containing information on the state or behavior of a physical system under an event that can be defined on a continuous or discrete space of time variation or spatial coordinates. In the broad sense, DSP systems mean a complex algorithmic, hardware and software. As a rule, systems contain specialized technical means of preliminary (or primary) signal processing and special technical means for secondary processing of signals. Means of pretreatment are designed to process the original signals observed in general case against a background of random noise and interference of a different physical nature and represented in the form of discrete digital samples, for the purpose of detecting and selection (selection) of the useful signal and evaluation characteristics of the detected signal.

A new method of digital signal processing in the frequency domain is proposed, using some properties of nonlinear orthogonal transformations. The theoretical bases of this method and the results of the statistical modeling, proving its efficacy in suppressing concentrated interference in the communication channels.
\end{abstract}

Introduction. Recent studies in digital technology have led to many existing revision previously views on possibility and feasibility of various complex signal processing algorithms, skipping the limitations of traditional approaches, obviously related to the properties of earlier analog components. For example, ordinary linear filtering, if carried out in the frequency domain, result in multiplying the current signal spectrum by the filter transfer function. However, when processing signals in digital form, you can use many other, including nonlinear spectral transformations, which do not correspond to any real analog filters. A typical example is homomorphic filtration and cepstral analysis, in which the logarithm of the spectrum is realized. Even this simple algebraic operation offers a great amount of information about signal processing. In this report, we consider a basic class of nonlinear transformations in the frequency domain and prove the effectiveness of their application in suppressing concentrated interference in communication channels.

Signals processing using nonlinear orthogonal transformations. Various orthogonal transforms-Fourier, Walsh, Haar, etc., are widely used in signal processing which represented in $y=U x$ where $\mathrm{U}$ is a unitary operator (for a discrete processing, a matrix), the inverse operator equal

$$
U^{-1}=U^{*}
$$

If $\mathrm{x}$ and $\mathrm{y}$ are elements of a Hilbert space, this mapping is represented as the corresponding integral transformation. The unitarily property can also be presented by non-linear operators, it remains as definition (1). They were first used in problems of nonlinear quantum mechanics [2] and, unlike linear unitary operators, in the general case they do not have integral representations similar to the Fourier transform.

The representation given by such an operator is described by a nonlinear differential equation of the Schrodinger type

$$
\frac{\partial \psi}{\partial \eta}=H(\psi) \psi
$$

with the boundary conditions $\psi\left(\eta_{0}\right)=x, \psi\left(\eta_{m}\right)=y$, where $\psi(\eta)=\psi(\eta, \lambda)$ function is of some Banach space that takes values in the Hilbert space of functions of $\lambda$ to which the elements $\mathrm{x}$ and $\mathrm{y}$ belong, and $H(\psi)-$ is a nonlinear continuous operator in this space $\eta, \eta_{m}$ is the initial and final values of the variable. The representation described by equations called operators with unitary nonlinearity. 
The use of non-linear orthogonal transformations (NOT) realizing such representation in digital signal processing problems, for the particular form of the operator $H(\psi)$,

$$
H(\psi)=-\alpha \frac{\partial}{\partial \lambda}-f(\psi),
$$

was first considered in $[3,4]$. The nonlinear orthogonal transformation $y=U(\psi) x$, described by equation (2) with such an operator $H(\psi)$, is realized as a result of linear $\left(\mathrm{G}_{\mathrm{k}}\right)$ and nonlinear $\left(\mathrm{N}_{\mathrm{k}}\right)$ operators

$$
U(\psi)=\prod_{k=1}^{n} G_{k} N_{k}\left[\psi_{k}\right] \cdot
$$

Recording type $U(\psi)$ means that the transformation $\mathrm{U}$ depends not only on the input signal $\mathrm{x}$, but also on the entire trajectory in a Hilbert space, described by equation (2) with such an input action. Each of the linear operators $G_{k}$ is an integral convolution type transformation with the Fresnel kernel

$$
g_{k}(\lambda)=g_{0 k} \exp \left\{i a_{k} \lambda^{2}\right\}
$$

where $\Delta \eta_{k}=\eta_{k+1}-\eta_{k}$ the step of digitizing by variable $\eta, n$ - number of pairs of links

$$
g_{0 k}=\sqrt{4 \pi \Delta \eta_{0} \alpha i}, a_{k}=1 / 4 \Delta \eta_{0} \alpha
$$

The nonlinear operators $N_{k}\left[\eta_{k}\right]$ correspond to the multiplication of the function $\psi_{k}(\lambda)=\psi\left(\eta_{k+1}, \lambda\right)$ by the transformation coefficients that depend on it

$$
N_{k}\left[\psi_{k}\right]=\exp \left\{\operatorname{if}\left(\psi_{k}\right)\right\}
$$

After a corresponding digitization with respect to the variable $\lambda$ these operators are easily realized in digital form. The transformation with the kernel (5) is realized using the FFT algorithm or the fast Fresnel transform.

The use of the described NSS allows solving a number of important problems of signal processing: compression of pulses in the time domain, selection of signals and impulse noise, spatial compression of image elements $[3,4]$. However, of particular interest is their use in the frequency domain, which has not yet been considered. In this case, the variable $\lambda$ in the above formulas should be interpreted as a frequency, and the described processing algorithm is preceded by a transition to the frequency domain using the FFT algorithm. Let us consider the practical application of such a transformation to suppress non-Gaussian focused jamming in communication channels.

Suppression of concentrated interference. The struggle with concentrated noise (SP) in communication channels is often hampered by the fact that their spectrum turns out to be commensurable in width with the signal spectrum. In this case, conventional methods of rejection filtering of interference inevitably lead to significant distortion of the signal and the loss of a large part of its energy. The efficiency of the notch filtering can be improved if the interference spectrum is preconditioned without significant change in the useful signal. Such an option is provided by the above-mentioned LVs: due to the nonlinearity property, they selectively act on the elements of the spectrum of the input mixture with different amplitudes and widths. With a certain choice of the parameter $\alpha$ and the function $f(\psi)$ in (3), the effect of selective compression of the concentrated noise and an increase in the efficiency of their subsequent rejection is provided. Distortions made in this case in the signal, if necessary, are eliminated by inverse transformation. It, by virtue of unitarity, is realized by a formula analogous to (4), with an inverse sequence of operators. The effectiveness of this method with respect to the reception of discrete messages was investigated by statistical computer modeling. In this case, a function was chosen in (3) $f(\psi)=k|\psi|^{2}$ and the optimal values of the parameters are chosen $\alpha$ and $k$.

Results of modeling and conclusions. In order to test the effectiveness of the proposed method for dealing with a DP was conducted statistical modeling receiving binary signals in a channel with white gaussian noise (WGN) and random CI, which is distributed over the squared amplitude Nakagami law duration

According to the truncated normal law, the phase - according to the uniform law. After suppression of the residual $\mathrm{CI}$ mixture was fed to the input of the correlation receiver.
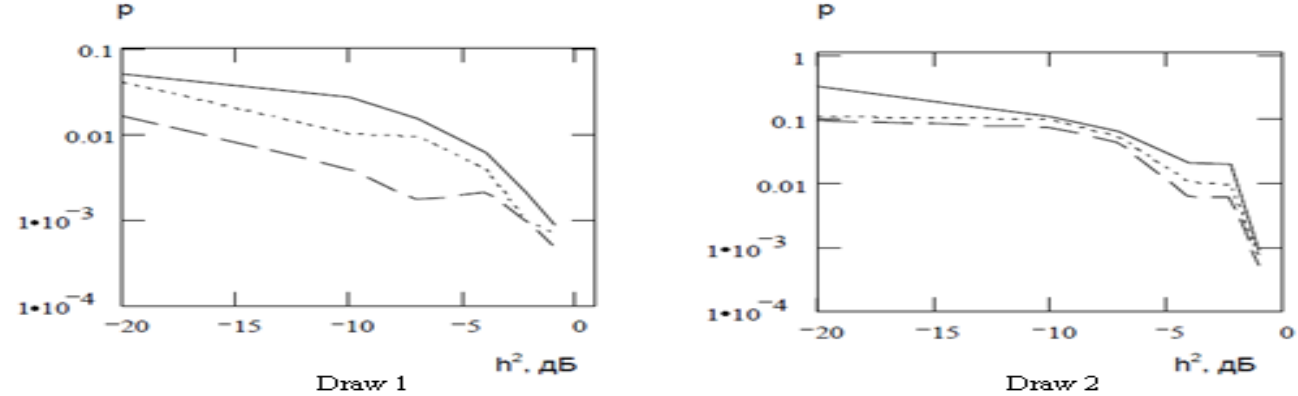
The results of the proposed method and 2 methods of linear filtration - a simple notch filter (SNF) with a rectangular AFC and an optimal Kolmogorov-Wiener filter (PCF). In Fig. 1 shows the dependence of the error probability on the signal-to-noise ratio for the proposed filter (dashed line), RF (solid line), PCF (dotted line) for $40 \%$ overlap of the spectra, in Fig. 2 - for $60 \%$. It can be seen that the proposed method of suppressing the SP with the reception of discrete messages yields a significant gain in comparison with other methods: even compared to the PCF at the input of the demodulator, by $8 \mathrm{~dB}$ at $40 \%$ overlap and by $2 \mathrm{~dB}$ at $60 \%$ overlap. Similar dependences obtained for different values of the dispersion of the amplitude of the CI spectrum show that it has little effect on the error probability. At the same time, the error probability depends more on the dispersion of the spectral width. This indicates that the efficiency of the proposed method can be improved by adapting to changes in signal characteristics and interference.

\section{References:}

1. Rao K.R. Discrete cosine transform / K.R. Rao, P.Yip. - San Diego : Academic press, 1990.

2. Ahmed N. Orthogonal transforms for digital signals processing / N.Ahmed, K.R. Rao. - Springer-Verlag, 1975.

3. Wang Z. Fast Algorithms for the discrete transform and for the discrete Fourier transform / Z.Wang // IEEE Tans, on Acombtics, Speech, and Signal Processing. - 1984. - Vol. 32, No. 4. - Pp. 803-816.

4. Chernyak V.M. PMD-Induced Fluctuations of Bit-Error Rate in Optical Fiber Systems / V.M. Chernyak et al. // Journal of lightwave technology. - 2004. - Vol. 22, No. 4. - Pp. 1155-1186.

5. Singer A.C. Electronic dispersion Compensation / A.C. Singer, N.R. Shanbhag, H.-M. Bae // IEEE Signal Processing Magazine. - 2008. -No. 11. - Pp. 119-130.

6. Anderson D. Variational a pproach to nonlinear pulse propagation in optical fibers / D.Anderson // Phys. Rev. Series : A Gen. Phys. - 1983. - Vol. 27, No. 6. - P $\rho$. 3135-3145.

7. Reflections. In New Directions in Time Series Analysis. Part I / by eds. D.Brillinger, P.Caines, J.Geweke, E.Parzen, M.Rosenblatt, M.Taqqu. - New York : Springer, 1992. - Pp. 387-389.

8. Brillinger D.R. The life and professional contributions of John W.Tukey / D.R. Brillinger // Ann. Statist. - 2002. Vol. 30. - Pp. 1535-1575.

9. Симончик К.К. Цифровая обработка сигналов : учеб. пособие / К.К. Симончик, А.Ю. Тропченко, М.В. Хитров. - СПб : СПбГУ ИТМО, 2012. - 108 с.

10. Turitsyn S.K. Variational approach to optical pulse propagation in dispersion compensated transmission systems / S.K. Turitsyn, I.Gabitov // Opt. Commun. - 1998. - Vol. 151. - Pp. 117-135.

\section{References:}

1. Rao, K.R. and Yip, P. (1990), Discrete cosine transform, Academic press, San Diego.

2. Ahmed, N. and Rao, K.R. (1975), Orthogonal transforms for digital signals processing, Springer-Verlag.

3. Wang, Z. (1984), «Fast Algorithms for the discrete transform and for the discrete Fourier transform», IEEE Tans, on Acombtics, Speech, and Signal Processing, Vol. 32, No. 4, pp. 803-816.

4. Chernyak, V.M. and others (2004), «PMD-Induced Fluctuations of Bit-Error Rate in Optical Fiber Systems», Journal of lightwave technology, Vol. 22, No. 4, pp. 1155-1186.

5. Singer, A.C., Shanbhag, N.R. and Bae, H.-M. (2008), «Electronic dispersion Compensation», IEEE Signal Processing Magazine, No. 11, pp. 119-130.

6. Anderson, D. (1983), «Variational a pproach to nonlinear pulse propagation in optical fibers», Phys. Rev., Series A Gen. Phys., Vol. 27, No. 6, pp. 3135-3145.

7. Brillinger, D., Caines, P., Geweke, J., Parzen, E., Rosenblatt, M. and Taqqu, M. (eds.) (1992), Reflections. In New Directions in Time Series Analysis, Part I, Springer, New York, pp. 387-389.

8. Brillinger, D.R. (2002), «The life and professional contributions of John W. Tukey», Ann. Statist., Vol. 30, pp. $1535-1575$.

9. Simonchik, K.K., Tropchenko, A.Ju. and Hitrov, M.V. (2012), Cifrovaja obrabotka signalov, SPbGU ITMO, Sankt-Peterburg, $108 \mathrm{p}$.

10. Turitsyn, S.K. and Gabito, I. (1998), «Variational approach to optical pulse propagation in dispersion compensated transmission systems», Opt. Commun., Vol. 151, pp. 117-135.

Ivanichenko Yevhen Victorovich - post-graduate student, senior lecturer at the Department of EnergyEfficient Technologies of the State University.

Scientific interests:

- renewable energy;

- alternative energy;

- information protection;

- automatic;

- automation of production. 